\title{
CURVATURE CHARACTERIZATIONS OF TWISTOR SPACES OVER FOUR-DIMENSIONAL RIEMANNIAN MANIFOLDS
}

\author{
BRENDAN FOREMAN $^{\dagger}$
}

\begin{abstract}
In this paper, we study the complex contact structure of a twistor space over a selfdual, Einstein 4-manifold with nonzero scalar curvature. Although the existence of such a structure has been known and well utilized by researchers for several decades now, the Hermitian geometry resulting from the complex contact structure is still in the process of being fully developed. Here we give a characterization of such twistor spaces as those satisfying a curvature (and hence purely geometric) identity. Later, we describe how this result fits in with other areas of research in complex contact geometry.
\end{abstract}

\section{Introduction}

The study of the twistor spaces over Riemannian four-manifolds began with the seminal results by Atiyah, Hitchin and Singer in [2] regarding the $S O(4)$ splitting of the Weyl curvature tensor. Following the heels of this paper, were the results found independently by Salamon ([23]) and Bérard-Bergery ([3]), which characterized the defined almost complex structures on the twistor spaces according to what was happening with the metric structure of the base manifold. In all three lines of research, the object was to encode the information of the downstairs manifold into the complex (or at least almost complex) geometry of the twistor space.

Since the publication of these three important papers, the field of twistor theory has exploded. Although for many researchers, the emphasis continues to be what information about the base manifold can be gleaned from the twistor space, many researchers have studied the twistor spaces as geometric objects of their own. Most notable for our purposes here are the curvature equations derived by Davidov and Muscarov in [8] and the famous Inverse Twistor Construction (see [22]). Although this theorem generalizes to any odd dimension for $Z$, this last theorem is stated by Swann in [24] as follows.

\section{THEOREM 1.1. Let $Z$ be a three-dimensional complex manifold such that}

1991 Mathematics Subject Classification: Primary 53T58.

${ }^{\dagger}$ Research supported by NSF grant \#DMS95-20481

Received April 5, 2001; revised May 27, 2002. 
1. There is a holomorphic line bundle $\mathscr{L}$ over $Z$ and a one-form $\theta \in \Omega^{1}(Z, \mathscr{L})$ such that $\theta \wedge d \theta$ vanishes nowhere, that is, $Z$ is a complex contact manifold;

2. The fibration $Z \rightarrow M$ is not holomorphic, but the fibres are complex lines with normal bundle 2O(1) and these lines are transverse to the contact distribution, that is, $\theta$ is nowhere zero on the vertical fibres; and

3. $Z$ admits a real structure $\sigma$, that is, an anti-holomorphic involution such that $\sigma$ preserves the vertical fibres and $\sigma^{*} \theta=\bar{\theta}$.

Then $Z$ is the twistor space over a four-dimensional self-dual, Einstein Riemannian manifold.

One aspect of twistor geometry that is still being developed is the rich Hermitian geometry that arises from the complex contact structure. There has been much work since the 1950s in this area regarding real contact geometry (see, for example, [5]), but the complex contact side of things is still being mapped out. In this paper, we will use this Hermitian geometry associated to the complex contact structure to prove the following theorem.

TheOREM 6.1. Let $(Z, J)$ be a three-dimensional manifold. Then $Z$ is biholomorphic to the twistor space of a self-dual, Einstein four-manifold with nonzero scalar curvature, if and only if $Z$ has a hermitian metric $h$ and a J-invariant splitting $T Z=\mathscr{V} \oplus \mathscr{H}$ such that $\operatorname{dim}_{\boldsymbol{R}} \mathscr{H}=4, \mathscr{V}$ is totally geodesic (and hence integrable), $\mathscr{H}$ is holomorphic with $h$ bundlelike through $\mathscr{V}$, and, for every unit $U \in \mathscr{V}$, the Riemannian curvature of $Z, R$, satisfies

$$
\begin{aligned}
R_{X Y} U= & \lambda u(Y) \mathscr{H} X+\mu v(Y) J^{\prime} X \\
& -\lambda u(X) \mathscr{H} Y-\mu v(X) J^{\prime} Y \\
& +\left[v_{1} h\left(J^{\prime} X, Y\right)+v_{2} h\left(J^{\prime \prime} X, Y\right)\right] V,
\end{aligned}
$$

where $V=-J U, u(E)=h(U, E), v(E)=h(V, E)$ and $J^{\prime}=J \circ \mathscr{H}$ and $J^{\prime \prime}=J \circ \mathscr{V}$ for some constants, $\lambda, \mu, v_{1}$ and $v_{2}$, such that $\lambda+\mu \neq 0$ and $v_{2}>0$.

The curvature identity in the theorem above is actually a generalization of what is called a "normal complex contact structure." This notion-derived from an analogue in real contact geometry - was first developed by Ishihara and Konishi in [17] but later expanded more satisfactorily by Korkmaz in [21]. More recently, some work has been done regarding another category of normal complex contact structures, of which the complex Heisenberg group is an element, in [10] and [11].

After a brief tour of complex contact geometry in the first section, we conduct a quick exposition of twistor theory over four-dimensional Riemannian manifolds, including a few well-known results. Next, restricting our attention to the twistor spaces over self-dual, Einstein four-manifolds with non-zero scalar curvature, we explore some aspects of their holomorphic structures, including their complex contact structures. This section ends with a very technical theorem detailing some Hermitian and curvature identities. In the fifth section, we 
detail some results regarding holomorphic subbundles of codimension one, so that we can prove the main theorem in the sixth section. Finally, in the last section, we discuss how this results fits within the current research in the Hermitian geometry of complex contact manifolds, giving us the ability to re-state the theorem in more aesthetically pleasing ways.

Unlike the seminal work by Davidov and Muscarov in [8], we will be using the standard wedge coefficients for forms in order to conform with the currently used terminology of complex contact geometry in [18], [21] and [11]. So, for example, if $\alpha$ and $\beta$ are vectors, then the 2 -vector $\alpha \wedge \beta$ is defined to be:

$$
\alpha \wedge \beta=\frac{1}{2}(\alpha \otimes \beta-\beta \otimes \alpha) .
$$

A particular consequence of this is that, for any vectors $E, F$ and 1 -form $\beta$,

$$
d \beta(E, F)=\frac{1}{2}(E \beta(F)-F \beta(E)-\beta([E, F])) .
$$

I thank David Blair of Michigan State University, Johann Davidov and Oleg Muscarov of the Bulgarian Academy of Sciences, and David Singer of Case Western Reserve University, all of whom provided valuable help and inspiration for this work.

\section{A brief introduction to complex contact geometry}

Although we will not be using too many of the ideas from this section, it will be useful to give a brief explanation of the current terms regarding complex contact geometry. Recall that a complex contact manifold is a complex manifold $(Z, J)$ of complex dimension $2 n+1$ such that there is an open atlas $\mathscr{U}=\{\mathcal{O}\}$ of $Z$ for which the following statements hold:

1. On each $\mathcal{O} \in \mathscr{U}$, there is a holomorphic 1-form $\eta$ such $\eta \wedge(d \eta)^{n} \neq 0$ everywhere on $\mathcal{O}$.

2. For $\mathcal{O}, \mathcal{O}^{\prime} \in \mathscr{U}$ with respective 1 -forms $\eta, \eta^{\prime}$, there is holomorphic function $f: \mathcal{O} \cap \mathcal{O}^{\prime} \rightarrow \boldsymbol{C}^{*}$ such that $\eta^{\prime}=f \eta$.

If we define $\mathscr{H}^{1,0}=\left(\bigcup_{\mathcal{O} \in \mathscr{U}} \operatorname{ker}(\eta)\right) \cap T^{1,0} Z$, then $\mathscr{H}^{1,0}$ is a well-defined, holomorphic subbundle of $T^{1,0} Z$ of maximal rank and complex dimension $2 n$, called the holomorphic contact subbundle of $Z$. Set $\mathscr{H}=\left\{X \in T^{R} Z: X-i J X \in \mathscr{H}^{1,0}\right\}$. Thus, $\mathscr{H}$ is a subbundle of $T Z$ with maximal rank, which is holomorphic in the sense that there are local bases of $\mathscr{H}\left\{X_{1}, \ldots, X_{4 n}\right\}$ such that $\mathscr{L}_{X_{j}} J=0$ for $j=$ $1, \ldots, 4 n$. We will call $\mathscr{H}$ the holomorphic contact subbundle or simply the contact subbundle.

Let $(Z, J)$ be a complex contact manifold with complex contact subbundle $\mathscr{H}$. We say that a set of $\boldsymbol{C}$-valued 1 -forms $\{\pi\}$ whose domains cover $Z$ is a normalized contact structure, if any two 1 -forms $\pi, \pi^{\prime}$ in this set with respective domains $\mathcal{O}, \mathcal{O}^{\prime}$ satisfy:

1. $\mathscr{H}=\operatorname{ker}(\pi)$ on $\mathcal{O}$.

2. On $\mathcal{O} \cap \mathcal{O}^{\prime}, \pi^{\prime}=f \pi$ for some $f: \mathcal{O} \cap \mathcal{O}^{\prime} \rightarrow S^{1}$. 
Note that for any $\pi$ in this set, $\pi=u-i v$ where $u$ and $v$ are real 1 -forms such that $v=u \circ J$. By taking a hermitian metric on the complex line bundle $L=$ $\left\{\omega \in \Lambda^{1,0} Z: \omega(\mathscr{H})=0\right\}$, it is fairly easy to construct a normalized contact structure on $Z$. This very construction shows that such structures are far from unique. However, they are handy objects to use because of the next theorem (See [9] for proof of all the following results).

THEOREM 2.1. Given a normalized contact structure $\{\pi\}$ on a complex contact manifold $Z$, there is a unique, two-real-dimensional, J-invariant subbundle $\mathscr{V}$ of $T Z$ such that

1. $T Z \cong \mathscr{H} \oplus \mathscr{V}$.

2. For any element $\pi=u-i v$ of the normalized contact structure, there is a local basis of $\mathscr{V},\{U, J U\}$ defined by:

(a) $u(U)=1, v(U)=0, u(J U)=0, v(J U)=-1$,

(b) $d u(U, X)=0, d v(J U, X)=0$ for any $X \in \mathscr{H}$.

Since there is a splitting $T Z \cong \mathscr{H} \oplus \mathscr{V}$, we have two natural projections $T Z \rightarrow \mathscr{H}$ and $T Z \rightarrow \mathscr{V}$. We will denote these respective projections by the same name as their corresponding subbundles, $\mathscr{H}$ and $\mathscr{V}$.

For a specific normalized contact structure, we define two new 2-forms, $\hat{G}$ and $\hat{H}$, by

$$
\begin{aligned}
\hat{G}(X, Y) & =d u(\mathscr{H} X, \mathscr{H} Y), \\
\hat{H}(X, Y) & =d v(\mathscr{H} X, \mathscr{H} Y) .
\end{aligned}
$$

Then it is not difficult to show that

$$
\begin{aligned}
& \hat{G}=d u-\sigma \wedge v, \\
& \hat{H}=d v+\sigma \wedge u,
\end{aligned}
$$

where $\sigma$ is a connection form of the bundle $L$. We call $\sigma$ the Ishihara-Konishi connection of the normalized contact structure and $d \sigma$ its Ishihara-Konishi curvature.

Given a normalized contact structure $\{\pi\}$, a hermitian metric $h$ is called associated to $\eta$, if it satisfies the following statements:

1. For any unit, vertical vector field $U \in \Gamma^{\infty}(\mathscr{V})$, the real endomorphism $k_{U}$ defined by

$$
k_{U}(X)=\operatorname{skew}\left(X \mapsto \mathscr{H} \nabla_{X} U\right)
$$

satisfies $k_{U}^{2}=-i d_{\mathscr{H}}, k_{J U}=J \circ k_{U}=-k_{U} \circ J$.

2. $\mathscr{V} \perp \mathscr{H}$.

Thus, the covariant derivatives $\nabla U$ and $\nabla(J U)$ of an associated metric induce a quaternionic structure $\left\{k_{U}, k_{J U}, J^{\prime}=J \circ \mathscr{H}\right\}$ on $\mathscr{H}$. Associated metrics were shown to exist in [18]. Note also that for any unit vertical vector $U$,

$$
d \sigma(U, J U)=\frac{1}{2} h\left(R_{U J U} J U, U\right) .
$$




\section{The twistor space of a four-dimensional Riemannian manifold}

Let $(M,<\rangle$,$) be a 4-dimensional Riemannian manifold. For any open set$ $U$ of $M$, there is an orthonormal, oriented basis $\left\{e_{1}, e_{2}, e_{3}, e_{4}\right\}$ of $T M$ with dual basis $\left\{e^{1}, e^{2}, e^{3}, e^{4}\right\}$ of $T^{*} M$.

On $\Lambda^{2}=\Lambda^{2}(T M)$, the Hodge star operator is an involution, i.e. $*^{2}=$ id. So, the eigenvalues of $*$ are +1 and -1 . In fact, the eigenspaces are locally given by:

$$
\Lambda_{+}^{2} M=\left\langle\bar{w}_{1}, \bar{w}_{2}, \bar{w}_{3}, \bar{w}_{4}\right\rangle, \quad \Lambda_{-}^{2} M=\left\langle w_{1}, w_{2}, w_{3}, w_{4}\right\rangle,
$$

where

$$
\begin{array}{ll}
w_{1}=e_{1} \wedge e_{2}-e_{3} \wedge e_{4} & \bar{w}_{1}=e_{1} \wedge e_{2}+e_{3} \wedge e_{4} \\
w_{2}=e_{1} \wedge e_{3}-e_{4} \wedge e_{2} & \bar{w}_{2}=e_{1} \wedge e_{3}+e_{4} \wedge e_{2} \\
w_{3}=e_{1} \wedge e_{4}-e_{2} \wedge e_{3} & \bar{w}_{3}=e_{1} \wedge e_{4}+e_{2} \wedge e_{3} .
\end{array}
$$

The inner product $\langle$,$\rangle on T M$ induces a metric on $\Lambda^{2} M$ by setting:

$$
\langle\alpha \wedge \beta, \gamma \wedge \delta\rangle=\frac{1}{2}\left|\begin{array}{cc}
\langle\alpha, \gamma\rangle & \langle\alpha, \delta\rangle \\
\langle\beta, \gamma\rangle & \langle\beta, \delta\rangle
\end{array}\right| \text { for primitive 2-vectors, }
$$

and defining bilinearly on general 2 -vectors.

The induced Levi-Civita connection on $\Lambda^{2} M$ from that of the original Riemannian metric $\langle$,$\rangle on T M$ is the bundle connection for this new metric on $\Lambda^{2} M$. Furthermore, taking $\left\{w_{1}, w_{2}, w_{3}\right\}$ as the oriented, orthonormal basis of $\Lambda_{-}^{2} M$, we have a well-defined cross-product on $\Lambda_{-}^{2} M$ with $w_{1} \times w_{2}=w_{3}$.

The Riemannian curvature $\tilde{R}$ of $(M,<\rangle$,$) induces an operator \mathscr{R}$ on $\Lambda^{2} M$ by

$$
\langle\mathscr{R}(X \wedge Y), Z \wedge W\rangle=-\left\langle\tilde{R}_{X Y} Z, W\right\rangle .
$$

Also, the bundle connection $\nabla$ on $\Lambda^{2} M$ induces a bundle curvature given by:

$$
R_{X \wedge Y}^{\nabla}(Z \wedge W)=\tilde{R}_{X \wedge Y} Z \wedge W+Z \wedge \tilde{R}_{X \wedge Y} W .
$$

It is well-known that the relationship between these curvatures on $\Lambda_{-}^{2}$ is given by:

$$
\left\langle R_{X Y}^{\nabla} \alpha, \beta\right\rangle=\langle\mathscr{R}(\alpha \times \beta), X \wedge Y\rangle,
$$

for $\alpha, \beta \in \Lambda_{-}^{2} \quad$ (see [8] for details).

With respect to the basis $\left\{\bar{w}_{1}, \bar{w}_{2}, \bar{w}_{3}, w_{1}, w_{2}, w_{3}\right\}$, we write $\mathscr{R}: \Lambda_{+}^{2} \oplus \Lambda_{-}^{2} \rightarrow$ $\Lambda_{+}^{2} \oplus \Lambda_{-}^{2}$ as a block matrix:

$$
\mathscr{R}=\left(\begin{array}{cc}
A_{1} & { }^{t} B \\
B & A_{2}
\end{array}\right),
$$

where $A_{1}$ and $A_{2}$ are $3 \times 3$ anti-symmetric matrices.

Recall the definition of the Weyl curvature tensor: 


$$
\begin{aligned}
\tilde{C}_{X Y} Z= & \tilde{R}_{X Y} Z \\
& +\frac{1}{n-2}(\langle\tilde{Q} X, Z\rangle Y-\langle\tilde{Q} Y, Z\rangle X+\langle X, Z\rangle \tilde{Q} Y-\langle Y, Z\rangle \tilde{Q} X) \\
& -\frac{\tau}{(n-1)(n-2)}(\langle X, Z\rangle Y-\langle Y, Z\rangle X) .
\end{aligned}
$$

Let $\mathscr{W}: \Lambda^{2} \rightarrow \Lambda^{2}$ be defined by

$$
\langle\mathscr{W}(X \wedge Y), Z \wedge W\rangle=-\left\langle\tilde{C}_{X Y} Z, W\right\rangle .
$$

It was proven by Atiyah, Hitchin and Singer in [2] that $\mathscr{W}$ preserves the fibration $\Lambda^{2}=\Lambda_{-} \oplus \Lambda_{+}$so that

$$
\mathscr{R}=\left(\begin{array}{cc}
\mathscr{W}_{+}+\frac{\tau}{6} \mathrm{id} & { }^{t} B \\
B & \mathscr{W}_{-}+\frac{\tau}{6} \mathrm{id}
\end{array}\right),
$$

where $\mathscr{W}_{+}$and $\mathscr{W}_{-}$are the restrictions of $\mathscr{W}$ on $\Lambda_{+}$and $\Lambda_{-}$, respectively, and the operator $\mathscr{B}$, given by

$$
\mathscr{B} \equiv\left(\begin{array}{cc}
0 & { }^{t} B \\
B & 0
\end{array}\right)
$$

is the traceless Ricci operator. We say $(M,\langle\rangle$,$) is self-dual, if \mathscr{W}_{-}=0$ and antiself-dual, if $\mathscr{W}_{+}=0$.

Set $Z M=\left\{\sum_{j=1}^{\dot{3}} a_{j} w_{j} \in \Lambda_{-}^{2}:\left(a_{1}\right)^{2}+\cdots+\left(a_{3}\right)^{2}=1\right\} \cong S^{2} . \quad Z$ is called the twistor space of $(M,\langle\rangle$,$) . Let \pi: Z \rightarrow M$ be the natural projection. Each element $z \in Z_{x} M$ defines an almost complex structure $S_{z}$ on $T_{x} M$ by:

$$
\left\langle S_{z} X, Y\right\rangle=2\langle z, X \wedge Y\rangle \text {. }
$$

Since the space of almost complex structures compatible with a given inner product and orientation of $\boldsymbol{R}^{4}$ is $S^{2}$, we see that $\pi^{-1}(x)$ is the space of all almost complex structures which are compatible with $\left(T_{x} M,\langle\rangle,\right)$ and which are oppositely oriented to the given orientation of $\left\{e_{1}, e_{2}, e_{3}, e_{4}\right\}$.

For $z \in Z$, set $\mathscr{V}_{z}=\operatorname{ker}\left(\pi_{*}\right)_{z}$. Note that, if $x=\pi(z)$, we can identify $\mathscr{V}_{z}$ with all elements of $\left(\Lambda_{-} M\right)_{x}$ which are orthogonal to $z$. So, if $s$ is a section of $Z$ and $X \in T_{x} M$, we have $\left\langle\nabla_{X} s, s\right\rangle=(1 / 2) X\langle s, s\rangle=0$, i.e. $\nabla_{X} s \in \mathscr{V}_{z} \quad(\nabla$ here is the bundle connection on $\Lambda^{2} M$ induced by the Riemannian connection of $\left.\langle\rangle,\right)$.

For $X \in T_{x} M$ and section $s$ of $Z$, set $X^{\mathscr{H}}=s_{*} X-\nabla_{X} s$ and $\mathscr{H}_{z}=$ $\left\{X^{\mathscr{H}}: X \in T_{x} M, s(x)=z\right\}$, or equivalently

$\mathscr{H}_{z}=\left\{s_{*} X: X \in T_{x} M, \quad s\right.$ a section of $Z$ such that $s(x)=z$ and $\nabla s=0$ at $\left.x\right\}$.

This definition gives us a splitting $T M=\mathscr{H} \oplus \mathscr{V}$. We denote the natural projections from $T M$ to the respective subbundles by the same letter:

$$
\mathscr{H}: T M \rightarrow \mathscr{H}, \quad \mathscr{V}: T M \rightarrow \mathscr{V} .
$$

Set $J^{\prime}=J \circ \mathscr{H}$ and $J^{\prime \prime}=J \circ \mathscr{V}$. 


\section{Almost complex and hermitian structures on twistor spaces}

\subsection{The almost complex structure}

We define an almost complex structure on $Z$ as follows. Let $z \in Z$. Define $J$ on $T_{z} Z$ by:

$$
J X= \begin{cases}X \times z & \text { if } X \in \mathscr{V}_{z} \\ \left(\operatorname{hor}_{z} \circ S_{z} \circ \pi_{*}\right) X & \text { if } X \in \mathscr{H}_{z} .\end{cases}
$$

LEMma 4.1. Let $z_{1}, z_{2} \in \pi^{-1}(p)$, then

$$
S_{z_{1}} \circ S_{z_{2}}=-S_{z_{1} \times z_{2}} .
$$

Proof. Without loss of generality, assume that $z_{1}=w_{1}$ and $z_{2}=w_{2}$, so that we need only show that $S_{w_{1}} \circ S_{w_{2}}=-S_{w_{3}}$. Using the definitions of $w_{1}, w_{2}$ and $w_{3}$, it is easily seen that, with respect to the basis $\left\{e_{1}, e_{2}, e_{3}, e_{4}\right\}$,

$$
\begin{aligned}
S_{w_{1}} & =\left(\begin{array}{cc|cc}
0 & -1 & 0 & 0 \\
1 & 0 & 0 & 0 \\
\hline 0 & 0 & 0 & 1 \\
0 & 0 & -1 & 0
\end{array}\right), S_{w_{2}}=\left(\begin{array}{cc|cc}
0 & 0 & -1 & 0 \\
0 & 0 & 0 & -1 \\
\hline 1 & 0 & 0 & 0 \\
0 & 1 & 0 & 0
\end{array}\right), \\
S_{w_{3}} & =\left(\begin{array}{cc|cc}
0 & 0 & 0 & -1 \\
0 & 0 & 1 & 0 \\
\hline 0 & -1 & 0 & 0 \\
1 & 0 & 0 & 0
\end{array}\right) .
\end{aligned}
$$

It is then easily verified that $S_{w_{1}} \circ S_{w_{2}}=-S_{w_{3}}$.

Then the following result is well-known (see [8]):

THEOREM 4.2. The almost complex structure $J$ is integrable if and only if $\langle$, is self-dual. Furthermore, suppose that $\langle$,$\rangle is self-dual, then \mathscr{H}$ is holomorphic if and only if $\langle$,$\rangle is also Einstein.$

\subsection{Hermitian geometry of a twistor space}

From this point on, we will assume that $\langle$,$\rangle is self-dual and Einstein. For$ $t \in \boldsymbol{R}-(0)$, define the pseudo-Riemannian metric $h_{t}$ on $Z$ by:

$$
h_{t}=\pi^{*}\langle,\rangle+t\langle,\rangle^{v},
$$

where $\langle,\rangle^{v}$ is the restriction of the bundle metric on $\mathscr{V}$. This metric is called the Salamon-Bérard-Bergery metric with vertical coefficient $t$. For convenience, we'll refer to these as $S B B$ metrics, and frequently the value for $t$ will be explicitly given or understood.

These metrics have many widely-known properties, the proofs and statements 
of which can be found in [8]. We will just list a few here. It should be noted that most of these properties hold true for the SBB metric of the twistor space of any four-dimensional Riemannian manifold, not necessarily only one that is selfdual and Einstein.

THEOREM 4.3. Let $Z$ be the twistor space over a self-dual, Einstein fourmanifold $(M,\langle\rangle$,$) with S B B$ metric $h_{t}$. Then the following statements are true.

1. $h_{t}$ is hermitian with respect to the complex structure $J$.

2. $\mathscr{V}$ is a totally geodesic foliation with respect to $h_{t}$.

3. Let $X$ be a horizontal vector in $T_{z} Z$ such that $\pi_{*}(X)=\tilde{X}$ and $W$ be any vertical vector field defined in an open neighborhood of $z$. Then, by identifying $\mathscr{V}_{z}$ with 2-vectors in $\left(\Lambda_{-}^{2} M\right)_{\mid \pi(z)}$ that are perpendicular to $z$,

$$
\mathscr{H}\left(\nabla_{X} W\right)_{z}=-\frac{1}{2} \operatorname{hor}_{z}\left(\left(\tilde{R}_{z}(J W) \tilde{X}\right)\right),
$$

where hor $_{z}: T_{x} M \rightarrow T_{z} Z$ is the horizontal lift.

\subsection{Complex contact geometry of a twistor space}

Suppose $t>0$ so that $h_{t}$ is a Riemannian metric. Let $U^{\prime}$ be any local vertical vector field on $Z$ such that $\left\langle U^{\prime}, U^{\prime}\right\rangle^{v}=1$. Set

$$
\begin{gathered}
U_{t}=\frac{1}{\sqrt{t}} U^{\prime}, \quad V_{t}=-J U_{t}, \\
u_{t}(E)=h_{t}\left(U_{t}, E\right), \quad v_{t}(E)=h_{t}\left(V_{t}, E\right) .
\end{gathered}
$$

Then, for $X, Y \in \mathscr{H}_{z}$ with $p=\pi(z), \tilde{X}=\pi_{*}(X), \tilde{Y}=\pi_{*}(Y)$,

$$
\begin{aligned}
2 d u_{t}(X, Y) & =-u_{t}([X, Y]) \\
& =-h_{t}\left(U_{t},[X, Y]\right) \\
& =h_{t}\left(U_{t}, \tilde{R}_{p}(X, Y) z\right) \\
& =t\left\langle\tilde{R}_{p}(X, Y) z, U_{t}\right\rangle \\
& =\sqrt{t}\left\langle\tilde{R}_{p}(X, Y) z, U^{\prime}\right\rangle \\
& =\sqrt{t}\left\langle\mathscr{R}\left(z \times U^{\prime}\right), X \wedge Y\right\rangle \\
& =\sqrt{t}\left(\frac{\tau}{6}\right)\left\langle z \times U^{\prime}, X \wedge Y\right\rangle \\
& =\sqrt{t}\left(\frac{\tau}{12}\right) 2\left\langle z \times U^{\prime}, X \wedge Y\right\rangle \\
& =\sqrt{t}\left(\frac{\tau}{12}\right)\left\langle S_{z \times U^{\prime}} X, Y\right\rangle .
\end{aligned}
$$

If we set $h_{t}\left(k_{U_{t}} X, Y\right)=d u_{t}(X, Y)$ and $k_{U_{t \mid \mathscr{V}}}=0$, then at $z \in Z, \quad k_{U_{t}}=$ 
$\sqrt{t}(\tau / 24) S_{z \times U^{\prime}}$. In particular, $k_{U_{t} \mid \mathscr{H}}^{2}=-\mathrm{id}_{\mid \mathscr{H}}$ if and only if $t=(24 / \tau)^{2}$. We also get the following relationship between $d v_{t}$ and $d u_{t}$.

Lemma 4.4. For $X, Y \in \mathscr{H}_{z}, d v_{t}(X, Y)=d u_{t}(J X, Y)$. Also, $k_{V_{t}}=k_{U_{t}} \circ J=$ $-J \circ k_{U_{t}}$. Finally, $k_{U_{t}} X=\nabla_{X} U_{t}$.

Proof. Let $p=\pi(z), \tilde{X}=\pi_{*}(X)$, and $\tilde{Y}=\pi_{*}(Y)$. From the above work, we have:

$$
\begin{aligned}
2 d v_{t}(X, Y) & =\sqrt{t}\left(\frac{\tau}{12}\right)\left\langle S_{z \times V^{\prime}} \tilde{X}, \tilde{Y}\right\rangle \\
& =\sqrt{t}\left(\frac{\tau}{12}\right)\left\langle S_{J^{\prime} \times z} \tilde{X}, \tilde{Y}\right\rangle \\
& =\sqrt{t}\left(\frac{\tau}{12}\right)\left\langle S_{\left(U^{\prime} \times z\right) \times z} \tilde{X}, \tilde{Y}\right\rangle \\
& =-\sqrt{t}\left(\frac{\tau}{12}\right)\left\langle S_{U^{\prime}} \tilde{X}, \tilde{Y}\right\rangle .
\end{aligned}
$$

Also, using the above work,

$$
\begin{aligned}
2 d u_{t}(J X, Y) & =\sqrt{t}\left(\frac{\tau}{12}\right)\left\langle S_{z \times U^{\prime}} \circ S_{z} \tilde{X}, \tilde{Y}\right\rangle \\
& =-\sqrt{t}\left(\frac{\tau}{12}\right)\left\langle S_{\left(z \times U^{\prime}\right) \times z} \tilde{X}, \tilde{Y}\right\rangle \\
& =-\sqrt{t}\left(\frac{\tau}{12}\right)\left\langle S_{U^{\prime}} \tilde{X}, \tilde{Y}\right\rangle .
\end{aligned}
$$

This proves the first equation as well as the relation $k_{V_{t}}=k_{U_{t}} \circ \mathrm{J}$.

Note that

$$
d u_{t}(X, J Y)=h_{t}\left(k_{U_{t}} X, J Y\right)=-h_{t}\left(J \circ k_{U_{t}} X, Y\right)
$$

as well as

$$
\begin{aligned}
2 d u_{t}(X, J Y) & =\sqrt{t}\left(\frac{\tau}{12}\right)\left\langle S_{z \times U^{\prime}} \tilde{X}, S_{z} \tilde{Y}\right\rangle \\
& =-\sqrt{t}\left(\frac{\tau}{12}\right)\left\langle S_{z} \circ S_{z \times U^{\prime}} \tilde{X}, \tilde{Y}\right\rangle \\
& =\sqrt{t}\left(\frac{\tau}{12}\right)\left\langle S_{z \times\left(z \times U^{\prime}\right)} \circ S_{z} \tilde{X}, \tilde{Y}\right\rangle \\
& =-\sqrt{t}\left(\frac{\tau}{12}\right)\left\langle S_{U^{\prime}} \circ S_{z} \tilde{X}, \tilde{Y}\right\rangle \\
& =2 h_{t}\left(k_{U_{t}} \circ J X, Y\right)
\end{aligned}
$$


Thus, $k_{U_{t}} \circ J=-J \circ k_{U_{t}}$. Finally, by its definition, $h_{t}$ is a bundlelike metric on $Z$ with respect to the fibration $\pi: Z \rightarrow M$, that is, $\left(\mathscr{L}_{U_{t}} h_{t}\right)(X, Y)=0$. This is equivalent to

$$
0=h_{t}\left(\nabla_{X} U_{t}, Y\right)+h_{t}\left(\nabla_{Y} U_{t}, X\right)
$$

Thus,

$$
\begin{aligned}
h_{t}\left(k_{U_{t}} X, Y\right) & =d u_{t}(X, Y) \\
& =\frac{1}{2}\left(X u_{t}(Y)-Y u_{t}(X)-u_{t}([X, Y])\right) \\
& =-\frac{1}{2} h_{t}\left(U_{t}, \nabla_{X} Y-\nabla_{X} Y\right) \\
& =\frac{1}{2} h_{t}\left(\nabla_{X} U_{t}, Y\right)-\frac{1}{2} h_{t}\left(\nabla_{Y} U_{t}, X\right) \\
& =h_{t}\left(\nabla_{X} U_{t}, Y\right)
\end{aligned}
$$

This proves the lemma.

All of these results allow us to prove the following proposition.

Proposition 4.5. Let $(M, g)$ be a 4-dimensional Riemannian manifold with twistor space $Z$. Then $(Z, J, \mathscr{H})$ is a complex contact manifold if and only if $g$ is self-dual and Einstein.

Proof. Clearly, we need only to show that if $\mathscr{H}$ is holomorphic, then $\mathscr{H}$ is a complex contact structure.

Fix $t>0$. Let $U$ be a local unit vertical vector field on $Z$ with respect to $h_{t}$. Let $u=u_{t}$ and $v=v_{t}$ be the 1 -forms as defined in the previous paragraph. Set

$$
\pi=u-i v(\neq 0) .
$$

Then $\mathscr{H}=\operatorname{ker}(\pi)$ so that $\pi \in \mathscr{V}^{*} \cap \mathscr{H}^{\perp}$. Note also that

$$
d \pi=d u-i d v
$$

which is nondegenerate on $\mathscr{H}$.

Also, $\pi\left(T^{0,1} M\right)=(0)$. Since $\mathscr{H}$ is holomorphic, there exists a local function $f$ such that the 1 -form $\omega=f \pi$ is holomorphic. Then

$$
\begin{aligned}
\omega \wedge d \omega & =(f \pi) \wedge(d f \wedge \pi+f d \pi) \\
& =f^{2} \pi \wedge d \pi .
\end{aligned}
$$

Since $d \pi$ is non-degenerate on $\mathscr{H}$, we know that $\pi \wedge d \pi$ (and hence $\omega \wedge d \omega$ ) is nowhere zero on its domain. This proves that $\mathscr{H}$ is a complex contact structure.

By combining the above result with the work from the previous paragraph, we get the following proposition. 
Proposition 4.6. Suppose that $(M, g)$ is a self-dual, Einstein manifold with scalar curvature $\tau$. Then the SBB metric $h_{t}$ is associated to the complex contact structure $\mathscr{H}$ if and only if $t=(24 / \tau)^{2}$.

From this point on, we will drop the subscripts of " $t$ " when they are easily understood. For any vertical, unit vector $U$, define the local 1-form $\sigma_{U}$ by

$$
\sigma_{U}(E)=-h\left(\nabla_{E} U, J U\right) .
$$

It is easily seen that $\sigma_{J U}=\sigma_{U}$ and that $d \sigma_{U}$ is a globally-defined 2-form on $Z$.

The following technical proposition has several identities that we will need for the main theorem. Note that the first and second are true for any associated metric of a complex contact manifold, whereas the rest are particular to the associated metric of twistor spaces. Recall that $J^{\prime}=J \circ \mathscr{H}$ and $J^{\prime \prime}=J \circ \mathscr{V}$.

Proposition 4.7. Let $U$ be a vertical vector field. Set $V=-J U$ and $\sigma=\sigma_{U}$. Let $u$ and $v$ be the 1-forms corresponding to $U$ and $V$ with respect to h. Then, for $E, F \in T Z$,

1. $h\left(k_{U} E, F\right)=(d u-\sigma \wedge v)(E, F)$.

2. $\nabla_{U} k_{U}=-\sigma(U) k_{J U}$.

3. $d \sigma(E, F)=\operatorname{th}\left(J^{\prime \prime} E, F\right)+(\tau / 24) h\left(J^{\prime} E, F\right)$.

4. $d \sigma(E, F)=(1 / 2) h\left(R_{E F} U, V\right)+t(\tau / 24)^{2} h\left(J^{\prime} E, F\right)$.

5. $h\left(R_{E F} U, V\right)=2 \operatorname{th}\left(J^{\prime \prime} E, F\right)+2(\tau / 24)(1-t(\tau / 24)) h\left(J^{\prime} E, F\right)$.

6. $\nabla_{J U} k_{U}=(\tau / 24)(1-2 t(\tau / 24)) J^{\prime}-\sigma(J U) k_{J U}$.

7. $\nabla_{U} J=(\tau / 24)(1-2 t(\tau / 24)) k_{J U}$.

Proof. Proof of 1. By definition, $h\left(k_{U} X, Y\right)=d u(X, Y)$ for two horizontal vectors $X$ and $Y$. Now suppose that $W=U$ or $V$ and $F=U, V$ or in $\mathscr{H}$. Then

$$
\begin{aligned}
2 d u(W, F) & =-u([W, F]) \\
& =-h\left(\nabla_{W} F, U\right)+h\left(\nabla_{F} W, U\right) \\
& =h\left(\nabla_{W} U, F\right)-h\left(\nabla_{F} U, W\right) \\
& =-\sigma(F) v(W)+\sigma(W) v(F) \\
& =2(\sigma \wedge v)(W, F) .
\end{aligned}
$$

This proves the first statement. by 1 ,

Proof of 2. Let $\tilde{k}_{U}$ be the 2-form given by $\tilde{k}_{U}(E, F)=h\left(k_{U} E, F\right)$. Then,

$$
d \tilde{k}_{U}=-d \sigma \wedge v+\sigma \wedge d v
$$

Now,

$$
3 d \tilde{k}_{U}(E, F, G)=h\left(\left(\nabla_{E} k_{U}\right) F, G\right)+h\left(\left(\nabla_{G} k_{U}\right) E, F\right)+h\left(\left(\nabla_{F} k_{U}\right) G, E\right) .
$$


So, for $Y, Z \in \mathscr{H}$,

$$
3(\sigma \wedge d v)(U, Y, Z)=h\left(\left(\nabla_{U} k_{U}\right) Y, Z\right)+h\left(\left(\nabla_{Z} k_{U}\right) U, Y\right)+h\left(\left(\nabla_{Y} k_{U}\right) Z, U\right) .
$$

Hence,

$$
\begin{aligned}
h\left(\left(\nabla_{U} k_{U}\right) Y, Z\right) & =(\sigma \wedge d v)(U, Y, Z)-h\left(\left(\nabla_{Z} k_{U}\right) U, Y\right)-h\left(\left(\nabla_{Y} k_{U}\right) Z, U\right) \\
& =\sigma(U) d v(Y, Z)-h\left(\nabla_{Z} U, k_{U} Y\right)-h\left(\nabla_{Y}\left(k_{U} Z\right), U\right) \\
& =\sigma(U) d v(Y, Z)-h\left(\nabla_{Z} U, k_{U} Y\right)+h\left(k_{U} Z, \nabla_{Y} U\right) \\
& =\sigma(U) d v(Y, Z)-h\left(k_{U} Z, k_{U} Y\right)+h\left(k_{U} Z, k_{U} Y\right) \\
& =\sigma(U) h\left(k_{V} Y, Z\right) \\
& =-\sigma(U) h\left(k_{J U} Y, Z\right) .
\end{aligned}
$$

This proves the second statement.

Proof of 3. Let $U$ be any unit vertical field with respect to $h_{t}$ and $X$ be a horizontal vector field. Set $V=-J U$. Since $\mathscr{V}$ is totally geodesic with respect to $h_{t}$,

$$
\begin{aligned}
0 & =h_{t}\left(R_{X U} U, V\right) \\
& =h_{t}\left(\nabla_{X} \nabla_{U} U, V\right)-h_{t}\left(\nabla_{U} \nabla_{X} U, V\right)-h_{t}\left(\nabla_{[X, U]} U, V\right) \\
& =h_{t}\left(\nabla_{X}(\sigma(U) U), V\right)-h_{t}\left(\nabla_{U}\left(\sigma(X) V+2 k_{U} X\right), V\right)-\sigma([X, U]) \\
& =X \sigma(U)-U \sigma(X)-\sigma([X, U]) \\
& =2 d \sigma(X, U) .
\end{aligned}
$$

So, we see that $d \sigma(\mathscr{V}, \mathscr{H})=0$.

Now, suppose that $X, Y \in \mathscr{H}_{z}$ with $p=\pi(z), \tilde{X}=\pi_{*}(X), \tilde{Y}=\pi_{*}(Y)$. Then, from the identities of Theorem 4.3, we get

$$
\begin{aligned}
2 d \sigma(X, Y)_{\mid z} & =X \sigma(Y)_{\mid z}-Y \sigma(X)_{\mid z}-\sigma([X, Y])_{\mid z} \\
& =X\left\langle D_{\pi_{*} Y} U, z \times U\right\rangle-Y\left\langle D_{\pi_{*} X} U, z \times U\right\rangle-\left\langle D_{\left[\pi_{*} X, \pi_{*} Y\right]} U, z \times U\right\rangle \\
& =\left\langle R^{\nabla}(\tilde{X}, \tilde{Y}) U, z \times U\right\rangle \\
& =\frac{\tau}{6}\left\langle(\tilde{X} \wedge \tilde{Y})^{-}, z\right\rangle \\
& =\frac{\tau}{12} h_{t}\left(J^{\prime} X, Y\right) .
\end{aligned}
$$

Finally, $t\langle,\rangle^{v}$ is the standard metric on $S^{2}$ with sectional curvature $\sqrt{t}$. So, for any vertical vectors $W_{1}, W_{2}$,

$$
d \sigma\left(W_{1}, W_{2}\right)=t\left\langle J W_{1}, W_{2}\right\rangle^{v} .
$$

Proof of 4. Let $E, F \in T Z$. Then 
CURVATURE CHARACTERIZATIONS OF TWISTOR SPACES

$$
\begin{aligned}
2 d \sigma(E, F)= & E \sigma(F)-F \sigma(E)-\sigma([E, F]) \\
= & E h\left(\nabla_{F} U, V\right)-F h\left(\nabla_{E} U, V\right)-h\left(\nabla_{[E, F]} U, V\right) \\
= & h\left(\nabla_{E} \nabla_{F} U, V\right)+h\left(\nabla_{F} U, \nabla_{E} V\right)-h\left(\nabla_{F} \nabla_{E} U, V\right) \\
& -h\left(\nabla_{E} U, \nabla_{F} V\right)-h\left(\nabla_{[E, F]} U, V\right) \\
= & h\left(R_{E F} U, V\right)+h\left(k_{U} F, k_{V} E\right)-h\left(k_{U} E, k_{V} F\right) \\
= & h\left(R_{E F} U, V\right)-h\left(k_{U} F, k_{J U} E\right)+h\left(k_{U} E, k_{J U} F\right) \\
= & h\left(R_{E F} U, V\right)+h\left(\left(k_{U} k_{J U}-k_{J U} k_{U}\right) E, F\right) \\
= & h\left(R_{E F} U, V\right)+2 t\left(\frac{\tau}{24}\right)^{2} h\left(J^{\prime} E, F\right)
\end{aligned}
$$

Proof of 5. This comes from combining statements 3. and 4 .

Proof of 6. By statement 1., for $Y, Z \in \mathscr{H}$,

$$
\begin{gathered}
h\left(\left(\nabla_{V} k_{U}\right) Y, Z\right)+h\left(\left(\nabla_{Y} k_{U}\right) Z, V\right)+h\left(\left(\nabla_{Z} k_{U}\right) V, Y\right) \\
=(-d \sigma \wedge v+\sigma \wedge d v)(V, Y, Z) .
\end{gathered}
$$

Now,

$$
\begin{aligned}
\mathrm{LHS} & =-h\left(\left(\nabla_{J U} k_{U}\right) Y, Z\right)+h\left(\nabla_{Y}\left(k_{U} Z\right), V\right)-h\left(k_{U} \nabla_{Z} V, Y\right) \\
& =-h\left(\left(\nabla_{J U} k_{U}\right) Y, Z\right)-h\left(k_{U} Z, \nabla_{Y} V\right)-h\left(k_{U} k_{V} Z, Y\right) \\
& =-h\left(\left(\nabla_{J U} k_{U}\right) Y, Z\right)+h\left(k_{U} Z, k_{J U} Y\right)+h\left(k_{U} k_{J U} Z, Y\right) \\
& =-h\left(\left(\nabla_{J U} k_{U}\right) Y, Z\right)-t\left(\frac{\tau}{24}\right)^{2} h(Z, J Y)+t\left(\frac{\tau}{24}\right)^{2} h(J Z, Y) \\
& =-h\left(\left(\nabla_{J U} k_{U}\right) Y, Z\right)-2 t\left(\frac{\tau}{24}\right)^{2} h(Z, J Y),
\end{aligned}
$$

whereas

$$
\begin{aligned}
\mathrm{RHS} & =-d \sigma \wedge v(Y, Z, V)+\sigma(V) d v(Y, Z) \\
& =-d \sigma(Y, Z)+\sigma(J U) h\left(k_{J U} Y, Z\right) \\
& =-\frac{\tau}{24} h(J Y, Z)+\sigma(J U) h\left(k_{J U} Y, Z\right) .
\end{aligned}
$$

Thus,

$$
-h\left(\left(\nabla_{J U} k_{U}\right) Y, Z\right)-2 t\left(\frac{\tau}{24}\right)^{2} h(Z, J Y)=-\frac{\tau}{24} h(J Y, Z)+\sigma(J U) h\left(k_{J U} Y, Z\right) .
$$

This proves the statement.

Proof of 7 . Note that by substituting " $J U$ " for " $U$ " in statement 6 , we get: 


$$
\nabla_{U} k_{J U}=-\frac{\tau}{24}\left(1-t \frac{\tau}{24}\right) J^{\prime}+\sigma(U) k_{U}
$$

It is easily seen that $\nabla_{U} J=\nabla_{U} J^{\prime}$. Then

$$
\begin{aligned}
\nabla_{U} J & =-\nabla_{U}\left(k_{J U} \circ k_{U}\right) \\
& =-\left(\nabla_{U} k_{J U}\right) k_{U}-k_{J U} \nabla_{U} k_{U} \\
& =-\left(-\frac{\tau}{24}\left(1-2 t \frac{\tau}{24}\right) J^{\prime}+\sigma(U) k_{U}\right) k_{U}-k_{J U}\left(-\sigma(U) k_{J U}\right) \\
& =\frac{\tau}{24}\left(1-2 t \frac{\tau}{24}\right) k_{J U}
\end{aligned}
$$

\section{Holomorphic subbundles of complex codimension one}

In this section, we will study some of the properties of a holomorphic subbundle with codimension one for a complex manifold. Throughout this section, we will assume that $(Z, J)$ is a complex manifold with $T Z=\mathscr{H} \oplus \mathscr{V}$ where both $\mathscr{H}$ and $\mathscr{V}$ are $J$-invariant and $\operatorname{dim}_{C} \mathscr{V}=1$.

Recall that $\mathscr{H}$ is holomorphic if and only if it is locally spanned by a set of infinitesimal automorphisms of $J$, i.e. there is a local basis $\left\{X_{1}, \ldots, X_{2 N}\right\}$ such that $\mathscr{L}_{X_{j}} J=0$ for each $j$. This is equivalent to the dual subbundle $\mathscr{V}^{*}$ being holomorphic, that is, there is a local holomorphic 1-form, $\omega$, such that $\mathscr{H}=\operatorname{ker}(\omega)$. Thus, $\mathscr{H}$ is holomorphic if and only if there is a complex-valued 1 -form $\omega$ such that

$$
\mathscr{H}=\operatorname{ker}(\omega), \quad \bar{\partial} \omega=0 .
$$

We will use this fact to show the following proposition.

Proposition 5.1. Let $(Z, J)$ be a complex manifold with J-invariant subbundle fibration $T Z=\mathscr{H} \oplus \mathscr{V}$. Let $u$ and $v=u \circ J$ be a pair of local 1-forms such that $\mathscr{H} \subset \operatorname{ker}(u) \cap \operatorname{ker}(v)$. If $\mathscr{H}$ is holomorphic, then, for $X, Y \in \mathscr{H}$,

$$
\begin{aligned}
& d u(X, Y)=-d u(J X, J Y) \\
& d v(X, Y)=d u(J X, Y) .
\end{aligned}
$$

Proof. Let $X$ and $Y$ be local holomorphic sections of $\mathscr{H}$, i.e. as explained above, $X, Y \in \mathscr{H}$ and $\mathscr{L}_{X} J=\mathscr{L}_{Y} J=0$. Then

$$
\begin{aligned}
d u(J X, J Y) & =J X u(J Y)-J Y u(J X)-u([J X, J Y]) \\
& =-u([J X, J Y]) \\
& =-u\left(J^{2}[X, Y]\right) \\
& =u([X, Y]) \\
& =-d u(X, Y) .
\end{aligned}
$$


Since $\mathscr{H}$ is spanned by a basis of holomorphic sections, we know that the first part of the proposition holds in general.

Similarly, using the same $X$ and $Y$ as described above, we have

$$
\begin{aligned}
d v(X, Y) & =X v(Y)-Y v(X)-v([X, Y]) \\
& =-v([X, Y]) \\
& =-u \circ J([X, Y]) \\
& =-u([J X, Y]) \\
& =d u(J X, Y) .
\end{aligned}
$$

This proves the proposition.

\section{Main theorem}

We are now in a suitable position to state and prove the main theorem.

TheOREM 6.1. Let $(Z, J)$ be a three-dimensional manifold. Then $Z$ is biholomorphic to the twistor space of a self-dual, Einstein four-manifold with non-zero scalar curvature, if and only if $Z$ has a hermitian metric $h$ and $a$ J-invariant splitting $T Z=\mathscr{V} \oplus \mathscr{H}$ such that $\operatorname{dim}_{R} \mathscr{H}=4, \mathscr{V}$ is totally geodesic (and hence integrable), $\mathscr{H}$ is holomorphic with $h$ bundlelike through $\mathscr{V}$, and, for every unit $U \in \mathscr{V}$, the Riemannian curvature of $Z, R$, satisfies

$$
\begin{aligned}
R_{X Y} U= & \lambda u(Y) \mathscr{H} X+\mu v(Y) J^{\prime} X \\
& -\lambda u(X) \mathscr{H} Y-\mu v(X) J^{\prime} Y \\
& +\left[v_{1} h\left(J^{\prime} X, Y\right)+v_{2} h\left(J^{\prime \prime} X, Y\right)\right] V,
\end{aligned}
$$

where $V=-J U, u(E)=h(U, E), v(E)=h(V, E)$ and $J^{\prime}=J \circ \mathscr{H}$ and $J^{\prime \prime}=J \circ \mathscr{V}$ for some constants, $\lambda, \mu, v_{1}$ and $v_{2}$, such that $\lambda+\mu \neq 0$ and $v_{2}>0$.

Proof. Let $Z$ be the twistor space of a four-dimensional self-dual, Einstein manifold $(M,\langle\rangle$,$) with scalar curvature \tau \neq 0$. Let $h=h_{t}$ be the SBB metric on $Z$ for some $t>0$. We will first prove a number of claims, which, when taken together, will give us the desired curvature condition.

Before we go on to the claims, recall that the O'Neill tensors $T$ and $A$ are defined by:

$$
\begin{aligned}
& A(E, F)=\mathscr{H} \nabla_{\mathscr{H} E} \mathscr{V} F+\mathscr{V} \nabla_{\mathscr{H} E} \mathscr{H} F, \\
& T(E, F)=\mathscr{H} \nabla_{\mathscr{V} E} \mathscr{V} F+\mathscr{V} \nabla_{\mathscr{V} E} \mathscr{H} F .
\end{aligned}
$$

Since $\mathscr{V}$ is totally geodesic, we know that $T=0$.

Claim 1. Suppose $X, Y \in \mathscr{H}$. Then $R_{X Y} U=2(\tau / 24)(1-t(\tau / 24)) h\left(J^{\prime} X, Y\right) V$. 
By one of O'Neill's equations, $h\left(R_{X Y} Z, U\right)=-h\left(\left(\nabla_{Z} A\right)(X, Y), U\right)$ for any $Z \in \mathscr{H}$. By 4.3, at $z \in Z, h\left(\left(\nabla_{Z} A\right)(X, Y), U\right)=t\left\langle\nabla_{Z} \mathscr{R}(X, Y), z \times U\right\rangle$. Furthermore, since $\mathscr{R}=$ constant(id) on $\Lambda_{-}, \nabla \mathscr{R}=0$. Thus, $h\left(R_{X Y} Z, U\right)=0$, or $\mathscr{H} R_{X Y} U=0$. The fifth equation of Proposition 4.7 gives the rest of the claim.

Claim 2. $R_{U V} U=2 t V$.

Since $\mathscr{V}$ is totally geodesic, we know that $\mathscr{H} R_{U V}=0$. Proposition 4.7, part 5 tells us then that $R_{U V} U=2 t V$.

CLAim 3. $\mathscr{H} R_{U Y} U=-t(\tau / 24)^{2} Y$ for $Y \in \mathscr{H}$.

$$
\begin{aligned}
\mathscr{H} R_{U Y} U & =\mathscr{H} \nabla_{U} \nabla_{Y} U-\mathscr{H} \nabla_{Y} \nabla_{U} U-\mathscr{H} \nabla_{[U, Y]} U \\
& =\mathscr{H} \nabla_{U}\left(k_{U} Y+\sigma(Y) U\right)+\mathscr{H} \nabla_{Y}(\sigma(U) J U)-\mathscr{H} k_{U}[U, Y] \\
& =\mathscr{H} \nabla_{U}\left(k_{U} Y\right)+\sigma(U) \mathscr{H} \nabla_{Y}(J U)-\mathscr{H} k_{U}\left(\nabla_{U} Y\right)+\mathscr{H} k_{U} \nabla_{Y} U \\
& =\mathscr{H}\left(\nabla_{U} k_{U}\right) Y+\sigma(U) k_{J U} Y+k_{U} k_{U} Y \\
& =-\sigma(U) k_{J U} Y+\sigma(U) k_{J U} Y-t\left(\frac{\tau}{24}\right)^{2} Y \\
& =-t\left(\frac{\tau}{24}\right)^{2} Y
\end{aligned}
$$

Claim 4. $\mathscr{H} R_{X V} U=(\tau / 24)(1-t(\tau / 24)) J X$ for $X \in \mathscr{H}$.

For $X, Z \in \mathscr{H}$,

$$
\begin{aligned}
h\left(R_{X V} U, Z\right)= & h\left(\nabla_{X} \nabla_{V} U-\nabla_{V} \nabla_{X} U-\nabla_{[X, V]} U, Z\right) \\
= & h\left(\nabla_{X}(\sigma(V) V), Z\right)-h\left(\nabla_{V}\left(\sigma(X) V+k_{U} X\right), Z\right) \\
& -h\left(\nabla_{\nabla_{X} V} U, Z\right)+h\left(\nabla_{\nabla_{V} X} U, Z\right) \\
= & \sigma(V) h\left(\nabla_{X} V, Z\right)-h\left(\nabla_{V}\left(k_{U} X\right), Z\right) \\
& -h\left(\nabla_{k_{V} X} U, Z\right)+h\left(k_{U} \nabla_{V} X, Z\right) \\
= & \sigma(V) h\left(k_{V} X, Z\right)-h\left(\left(\nabla_{V} k_{U}\right) X, Z\right)-h\left(k_{U} k_{V} X, Z\right) \\
= & \sigma(J U) h\left(k_{J U} X, Z\right)+h\left(\left(\nabla_{J U} k_{U}\right) X, Z\right)+h\left(k_{U} k_{J U} X, Z\right) \\
= & \sigma(J U) h\left(k_{J U} X, Z\right)+h\left(\frac{\tau}{24}\left(1-2 t \frac{\tau}{24}\right) J X, Z\right)-\sigma(J U) h\left(k_{J U} X, Z\right) \\
& +t\left(\frac{\tau}{24}\right)^{2} h\left(J^{\prime} X, Z\right) \\
= & h\left(\frac{\tau}{24}\left(1-t \frac{\tau}{24}\right) J^{\prime} X, Z\right)
\end{aligned}
$$


Combining Claims 1 through 4, we get

$$
\begin{aligned}
R_{X Y} U= & t\left(\frac{\tau}{24}\right)^{2} u(Y) \mathscr{H} X+\frac{\tau}{24}\left(1-t \frac{\tau}{24}\right) v(Y) J^{\prime} X \\
& -t\left(\frac{\tau}{24}\right)^{2} u(X) \mathscr{H} Y-\frac{\tau}{24}\left(1-t \frac{\tau}{24}\right) v(X) J^{\prime} Y \\
& +\left[2 \frac{\tau}{24}\left(1-t\left(\frac{\tau}{24}\right)\right) h\left(J^{\prime} X, Y\right)+2 \operatorname{th}\left(J^{\prime \prime} X, Y\right)\right] V .
\end{aligned}
$$

In this context, the coefficients $\mu$ and $\lambda$ as indicated in the main theorem are given by:

$$
\begin{aligned}
& \lambda=t\left(\frac{\tau}{24}\right)^{2}, \\
& \mu=\frac{\tau}{24}\left(1-t \frac{\tau}{24}\right),
\end{aligned}
$$

so that $\lambda+\mu=\tau / 24 \neq 0$.

We now suppose that $Z$ satisfies the given curvature identity. Since $h\left(R_{J U U} U, J U\right)=1$, the leaves of the foliation $\mathscr{V}$ foliate 2 -spheres in $Z$. By Theorem 4.4 in [13] and the fact that $S^{2}$ is simply-connected, $Z$ fibres over a 4-manifold $M=Z / \mathscr{V}$. Let $\pi$ be the projection from $Z$ to $M$. The metric $h$ is bundle-like with respect to $\mathscr{V}$ on $Z$, so that there is a Riemannian metric $g$ on $M$ such that the projection $\pi:(Z, h) \rightarrow(M, g)$ is a Riemannian submersion.

Let $Z_{g}$ be the twistor space of $(M, g)$ with projection $\pi_{g}: Z_{g} \rightarrow M$. Viewing $Z_{g}$ as the space of almost complex structures on $M$, which are hermitian with respect to $g$, we define a map $\phi: Z \rightarrow Z_{g}$ as follows.

For $z \in Z$ and $x=\pi(z)$, let $\phi(z) \in\left(\pi_{g}\right)^{-1}(x)$ be the almost complex structure on $T_{x} M$ given by:

$$
\phi(z) X=\left(\pi_{*} \circ J \circ \operatorname{hor}_{z}\right)(X) \text { for each } X \in T_{x} M,
$$

where hor $_{z}: T_{x} M \rightarrow \mathscr{H}_{z}$ is the horizontal lift.

Since all of the maps involved with the definition of $\phi$ are clearly continuous, $\phi$ is a continuous map. Also, $\phi$ maps the vertical leaves of $Z$ to the vertical leaves of $Z_{g}$, that is, restricted to a vertical leaf of $Z, \phi$ is simply a continuous map from $S^{2}$ to $S^{2}$. Thus, if we can show that $\phi$ is locally one-to-one, i.e. an immersion, we will have proven the theorem.

Let $x \in M, z \in \pi^{-1}(x)$. Let $U$ be a unit vector in $\mathscr{V}_{z}$ and extend it as a geodesic vector field about a neighborhood of $z$. Let $t \mapsto \gamma(t) \in \pi^{-1}(x)$ be the unique geodesic such that $\gamma(0)=z$ and $\gamma_{*}(0)=U_{z}$. Since $\phi(\gamma(t))=\pi_{*} \circ J \circ$ hor $_{\gamma(t)} \in Z_{g}$, we know that $\phi_{*}(U) \neq 0$ if and only if

$$
\frac{d}{d t}\left(\left(\pi_{*} \circ J \circ \operatorname{hor}_{\gamma(t)}\right) X\right)_{t=0} \neq 0 \text { for any nonzero } X \in T_{x} M \text {. }
$$


Fix a nonzero $X \in T_{x} M$. Let $\left\{\psi_{s}\right\}$ be the local family of isometries which generate $U$ (so that $\gamma(t)=\psi_{t}(z)$ ). In order to continue, we will need some very technical results derived from the curvature identity.

Let $V=-J U$. Set $k_{U} E=\mathscr{H} \nabla_{E} U$, so that $d u(X, Y)=h\left(k_{U} X, Y\right)$ for any $X, Y \in \mathscr{H}$, where the 1 -form $u$ is defined by $u(E)=h(U, E)$. Also, set $\sigma(E)=$ $-h\left(\nabla_{E} U, J U\right)$

Since $h$ is a bundlelike metric, $k_{U}$ is skew-symmetric with respect to $h$. Also, the first equation of Proposition 5.1 tells us that $k_{U} \circ J=-J \circ k_{U}$, whereas the second tells us that $k_{J U}=J \circ k_{U}$.

Claim. $\mathscr{L}_{U} J=((\lambda+\mu) / \lambda) k_{J U}$ on $\mathscr{H}$.

Proof of Claim. Using the fact that $\mathscr{V}$ is totally geodesic, for $Y, Z \in \mathscr{H}$,

$$
\begin{aligned}
-\lambda h(Y, Z) & =h\left(R_{U Y} U, Z\right) \\
& =h\left(\nabla_{U} \nabla_{Y} U-\nabla_{Y} \nabla_{U} U-\nabla_{[U, Y]} U, Z\right) \\
& =h\left(\nabla_{U}\left(k_{U} Y\right)-\nabla_{Y}(\sigma(U) V)-\nabla_{\nabla_{U} Y} U+\nabla_{\nabla_{Y} U} U, Z\right) \\
& =h\left(\nabla_{U}\left(k_{U} Y\right)-\sigma(U) \nabla_{Y} V-k_{U}\left(\nabla_{U} Y\right)+k_{U}^{2} Y, Z\right) \\
& =h\left(\left(\nabla_{U} k_{U}\right) Y+\sigma(U) k_{J U} Y, Z\right)+h\left(k_{U}^{2} Y, Z\right) .
\end{aligned}
$$

Since the transformation $\nabla_{U} k_{U}+\sigma(U) k_{J U}$ is skew-symmetric and $k_{U}^{2}+$ id is symmetric (with respect to $h$ ),

$$
\nabla_{U} k_{U}+\sigma(U) k_{J U}=0, \quad k_{U}^{2}=-\lambda \text { id on } \mathscr{H} .
$$

Note that this also implies that $k_{U} \circ k_{J U}=-k_{J U} \circ k_{U}=\lambda J^{\prime}$.

Similarly,

$$
\begin{aligned}
-\mu h(J X, Z) & =h\left(R_{X J U} U, Z\right) \\
& =h\left(\nabla_{X} \nabla_{J U} U-\nabla_{J U} \nabla_{X} U-\nabla_{[X, J U]} U, Z\right) \\
& =h\left(\nabla_{X}(-\sigma(J U) J U)-\nabla_{J U}\left(k_{U} X\right)-\nabla_{\nabla_{X}(J U)} U+\nabla_{\nabla_{J U} X} U, Z\right) \\
& =-\sigma(J U) h\left(\nabla_{X}(J U), Z\right)-h\left(\left(\nabla_{J U} k_{U}\right) X, Z\right)-h\left(k_{U} \circ k_{J U} X, Z\right) \\
& =-\sigma(J U) h\left(k_{J U} X, Z\right)-h\left(\left(\nabla_{J U} k_{U}\right) X, Z\right)-\lambda h(J X, Z) .
\end{aligned}
$$

Thus, $\nabla_{J U} k_{U}=-\sigma(J U) k_{J U}-(\mu-\lambda) J^{\prime}$, or

$$
\nabla_{U} k_{J U}=\sigma(U) k_{U}+(\mu-\lambda) J^{\prime} .
$$

Then,

$$
\begin{aligned}
\lambda \nabla_{U} J & =-\nabla_{U}\left(k_{J U} \circ k_{U}\right) \\
& =-\left(\nabla_{U} k_{J U}\right) \circ k_{U}-k_{J U} \circ\left(\nabla_{U} k_{U}\right) \\
& =-\left(\sigma(U) k_{U}+2(\mu-\lambda) J^{\prime}\right) \circ k_{U}-k_{J U} \circ\left(-\sigma(U) k_{J U}\right) \\
& =(\mu-\lambda) k_{J U} .
\end{aligned}
$$


Now, suppose that $X$ is a horizontal, projectable vector field. Then

$$
\begin{aligned}
\mathscr{H}\left(\mathscr{L}_{U} J\right) X & =\mathscr{H}([U, J X]-J[U, X]) \\
& =\mathscr{H} \nabla_{U}(J X)-\mathscr{H} \nabla_{J X} U-J^{\prime} \nabla_{U} X+J^{\prime} \nabla_{X} U \\
& =\mathscr{H}\left(\nabla_{U} J\right) X-\mathscr{H} \nabla_{J X} U+J^{\prime} \nabla_{X} U \\
& =\left(\frac{\mu-\lambda}{\lambda}\right) k_{J U}-k_{U} J X+J k_{U} X \\
& =\left(\frac{\mu-\lambda}{\lambda}+2\right) k_{J U} X \\
& =\left(\frac{\lambda+\mu}{\lambda}\right) k_{J U} X .
\end{aligned}
$$

This proves the claim.

Now, continuing with the proof of the main theorem,

$$
\begin{aligned}
\frac{d}{d t}\left(\left(\pi_{*} \circ J \circ \operatorname{hor}_{\gamma(t)}\right) X\right)_{t=0} & =\frac{d}{d t}\left(\left(\pi_{*} \circ J \circ\left(\psi_{-t}\right)_{*} \circ \operatorname{hor}_{\gamma(0)}\right) X\right)_{t=0} \\
& =\lim _{t \rightarrow 0} \frac{1}{t}\left[\left(\pi_{*} \circ J \circ\left(\psi_{-t}\right)_{*} \circ \operatorname{hor}_{\gamma(0)}\right) X-\left(\pi_{*} \circ J \circ \operatorname{hor}_{\gamma(0)}\right) X\right] \\
& =\lim _{t \rightarrow 0} \frac{1}{t}\left[\pi_{*} \circ\left(\left(\psi_{t}\right)_{*} J \circ\left(\psi_{-t}\right)_{*}-J\right) \circ \operatorname{hor}_{\gamma(0)} X\right] \\
& =\left(\pi_{*} \circ \mathscr{L}_{U} J \circ \operatorname{hor}_{\gamma(0)}\right) X \\
& =\left(\frac{\lambda+\mu}{\lambda}\right)\left(\pi_{*} \circ k_{J U} \circ \operatorname{hor}_{\gamma(0)}\right) X \\
& \neq 0 .
\end{aligned}
$$

Thus, $\phi_{*}(U) \neq 0$, and that proves the theorem.

\section{Remarks}

The main theorem had its origin in a desire to understand the lowdimensional twistor spaces from a complex contact perspective. This had already been achieved to some extent in the higher dimensions by Theorem 6.1 in [11], which we restate here.

THEOREM 7.1. Let $M$ be a complex contact manifold with contact subbundle $\mathscr{H}$ and normalized contact structure $\{\pi=u-i v\} . \quad$ Let $T M=\mathscr{H} \oplus \mathscr{V}$ be the corresponding splitting with local vertical vector fields $\{U, V\}$ corresponding to the real forms $u$ and $v$. Suppose $g$ is an associated metric on $M$. Then $(M, g)$ is isometric to a twistor space for a quaternionic-Kähler manifold with positive scalar curvature if and only if 


$$
\begin{aligned}
& R_{X Y} U=u(Y) X-u(X) Y+v(Y) J X-v(X) J Y+2 g(J X, Y) V, \\
& R_{X Y} V=-v(Y) X+v(X) Y-u(Y) J X+u(X) J Y-2 g(J X, Y) U .
\end{aligned}
$$

This theorem and the main theorem of this paper both spring from two current topics in complex contact geometry: normal complex contact manifolds and fibrated complex contact manifolds.

\subsection{Normal complex contact manifolds}

The first subject area that motivates Theorem 6.1 is that of normal complex contact manifolds. Modelled after the original definition in the real contact category, a normal complex contact manifold is a complex contact manifold $Z$ with associated metric $h$ such that, for every unit $U \in \mathscr{V}$, and any $X, Y, Z \in T Z$,

$$
\begin{aligned}
h\left(R_{X Y} U, Z\right)= & u(Y) h(X, Z)-v(Y)(h(J X, Z)-d \sigma(X, Z)) \\
& -u(X) h(Y, Z)+v(X)(h(J Y, Z)-d \sigma(Y, Z)) \\
& +v(Z)\left[-2 h\left(J^{\prime} X, Y\right)+d \sigma(X, Y)-d \sigma\left(k_{U} X, k_{U} Y\right)\right] V .
\end{aligned}
$$

By setting $d \sigma^{\sharp}$ to be the $(1,1)$-tensor defined by $h\left(d \sigma^{\sharp}(E), F\right)=d \sigma(E, F)$, we can rewrite this definition as follows:

$$
\begin{aligned}
R_{X Y} U= & u(Y) X-v(Y) J X-u(X) Y+v(X) J Y \\
& +v(Y) d \sigma^{\sharp}(X)-v(X) d \sigma^{\sharp}(Y) \\
& +\left[-2 h\left(J^{\prime} X, Y\right)+d \sigma(X, Y)-d \sigma\left(k_{U} X, k_{U} Y\right)\right] V .
\end{aligned}
$$

This concept was originally due to Ishihara and Konishi in [17] but was recently redefined by Korkmaz in [21]. Using this definition, we can rewrite Theorem 6.1 as follows.

THEOREM 7.2. A three-dimensional complex manifold $(Z, J)$ is biholomorphic to the twistor space of a self-dual, Einstein four-manifold with non-zero scalar curvature if and only if $Z$ is a normal complex contact manifold such that the Ishihara-Konishi curvature $d \sigma$ is non-degenerate on $T Z$ and positive on $\mathscr{V}$.

Proof. First, we show that the SBB metrics such that $t=(\tau / 24)^{-2}$, i.e. those which are associated to the complex contact structure, are normal. Note that Proposition 4.7 already tells us that $d \sigma$ is non-degenerate on $T Z$ and positive on $\mathscr{V}$.

Using the proof of Theorem 6.1, we see that, for an SBB metric such that $t=(\tau / 24)^{-2}$,

$$
\begin{aligned}
R_{X Y} U= & u(Y) \mathscr{H} X+\left(\frac{\tau}{24}-1\right) v(Y) J^{\prime} X-u(X) \mathscr{H} Y-\left(\frac{\tau}{24}-1\right) v(X) J^{\prime} Y \\
& +2\left[\left(\frac{\tau}{24}-1\right) h\left(J^{\prime} X, Y\right)+\left(\frac{\tau}{24}\right)^{-2} h\left(J^{\prime \prime} X, Y\right)\right] V .
\end{aligned}
$$


Now, we use Proposition 4.7 to show that the right-hand side of the definition of normality gives us the same expression as above. The following computation uses two crucial facts. First, $v=u \circ J$, so that $v \circ J=-u$. Also, we can rewrite the vertical bundle metric $h\left(J^{\prime \prime} *, *\right)$ by $h\left(J^{\prime \prime} E, F\right)=$ $-2(u \wedge v)(E, F)$.

$$
\begin{aligned}
& \text { RHS }=u(Y) X-v(Y) J X-u(X) Y+v(X) J Y \\
& +v(Y)\left(\left(\frac{\tau}{24}\right)^{-2} J^{\prime \prime} X+\frac{\tau}{24} J^{\prime} X\right)-v(X)\left(\left(\frac{\tau}{24}\right)^{-2} J^{\prime \prime} Y+\frac{\tau}{24} J^{\prime} Y\right) \\
& +\left[-2 h\left(J^{\prime} X, Y\right)+\left(\frac{\tau}{24}\right)^{-2} h\left(J^{\prime \prime} X, Y\right)+\frac{\tau}{24} h\left(J^{\prime} X, Y\right)\right. \\
& \left.-\frac{\tau}{24} h\left(J^{\prime} k_{U} X, k_{U} Y\right)\right] V \\
& =u(Y) X+v(Y)\left(-J X+\left(\frac{\tau}{24}\right)^{-2} J^{\prime \prime} X+\frac{\tau}{24} J^{\prime} X\right) \\
& -u(X) Y-v(X)\left(-J Y+\left(\frac{\tau}{24}\right)^{-2} J^{\prime \prime} Y+\frac{\tau}{24} J^{\prime} Y\right) \\
& +\left[-2 h\left(J^{\prime} X, Y\right)+\left(\frac{\tau}{24}\right)^{-2} h\left(J^{\prime \prime} X, Y\right)+2 \cdot \frac{\tau}{24} h\left(J^{\prime} X, Y\right)\right] V \\
& =u(Y) \mathscr{H} X+u(Y) v(X) V+v(Y)\left(-J^{\prime} X+\frac{\tau}{24} J^{\prime} X\right) \\
& +v(Y) v\left(-J X+\left(\frac{\tau}{24}\right)^{-2} J^{\prime \prime} X\right) V \\
& -u(X) \mathscr{H} Y-u(X) v(Y) V-v(X)\left(-J^{\prime} Y+\frac{\tau}{24} J^{\prime} Y\right) \\
& -v(X) v\left(-J Y+\left(\frac{\tau}{24}\right)^{-2} J^{\prime \prime} Y\right) V \\
& {\left[2\left(\frac{\tau}{24}-1\right) h\left(J^{\prime} X, Y\right)+\left(\frac{\tau}{24}\right)^{-2} h\left(J^{\prime \prime} X, Y\right)\right] V} \\
& =u(Y) \mathscr{H} X+u(Y) v(X) V+\left(\frac{\tau}{24}-1\right) v(Y) J^{\prime} X-\left(\left(\frac{\tau}{24}\right)^{-2}-1\right) v(Y) u(X) V
\end{aligned}
$$




$$
\begin{aligned}
& -u(X) \mathscr{H} Y-u(X) v(Y) V-\left(\frac{\tau}{24}-1\right) v(X) J^{\prime} Y \\
& +\left(\left(\frac{\tau}{24}\right)^{-2}-1\right) v(X) u(Y) V \\
& {\left[2\left(\frac{\tau}{24}-1\right) h\left(J^{\prime} X, Y\right)+\left(\frac{\tau}{24}\right)^{-2} h\left(J^{\prime \prime} X, Y\right)\right] V } \\
= & u(Y) \mathscr{H} X+\left(\frac{\tau}{24}-1\right) v(Y) J^{\prime} X-u(X) \mathscr{H} Y-\left(\frac{\tau}{24}-1\right) v(X) J^{\prime} Y \\
& 2\left[\left(\frac{\tau}{24}-1\right) h\left(J^{\prime} X, Y\right)+\left(\frac{\tau}{24}\right)^{-2} h\left(J^{\prime \prime} X, Y\right)\right] V .
\end{aligned}
$$

Thus, every associated SBB metric is a normal complex contact metric.

We now assume that $(Z, h)$ is a normal complex contact manifold. From page 1357 of [21], we know that

$$
\begin{aligned}
h\left(\left(\nabla_{E} J\right) F, G\right)= & -u(E)\left(d \sigma\left(G, k_{U} F\right)-2 h\left(k_{J U} F, G\right)\right) \\
& +v(E)\left(d \sigma\left(G, k_{J} F\right)-2 h\left(k_{U} F, G\right)\right) .
\end{aligned}
$$

In particular, $h\left(\left(\nabla_{U} J\right) Y, Z\right)=d \sigma\left(Z, k_{U} Y\right)-2 h\left(k_{J U} Y, Z\right)$. Given that $k_{U} \circ k_{J U}=$ $-k_{J U} \circ k_{U}$, it is easily seen that $\mathscr{H}\left(\nabla_{U} J\right) k_{U}=k_{U}\left(\nabla_{U} J\right) \mathscr{H}$. Also, $(\nabla J) J=$ $-J(\nabla J)$. Combining both of these facts with the above identity, we find that, for any $X, Y \in T Z$,

$$
\begin{aligned}
d \sigma\left(k_{U} X, k_{U} Y\right) & =-d \sigma(\mathscr{H} X, \mathscr{H} Y), \\
d \sigma(J X, J Y) & =-d \sigma(X, Y) .
\end{aligned}
$$

Now, since $\operatorname{dim}_{\boldsymbol{R}} \mathscr{H}=4, \operatorname{dim}_{\boldsymbol{R}} \mathscr{V}=2, d \sigma$ is nondegenerate on $\mathscr{H}$ and positive on $\mathscr{V}$, we know that, for any $X, Y \in T Z$,

$$
d \sigma(X, Y)=\alpha_{1} h\left(J^{\prime} X, Y\right)+\alpha_{2} h\left(J^{\prime \prime} X, Y\right),
$$

for some $\alpha_{1} \neq 0$ and $\alpha_{2}>0$. Substituting this into the definition for normality and simplifying, we get that, for any $X, Y \in T Z$,

$$
\begin{aligned}
R_{X Y} U= & u(Y) \mathscr{H} X+\left(\alpha_{1}-1\right) v(Y) J^{\prime} X \\
& -u(X) \mathscr{H} Y-\left(\alpha_{1}-1\right) v(X) J^{\prime} Y \\
& +\left[2\left(\alpha_{1}-1\right) h\left(J^{\prime} X, Y\right)+2 \alpha_{2} h\left(J^{\prime \prime} X, Y\right)\right] V .
\end{aligned}
$$

With $\lambda=1, \mu=\alpha_{1}-1, v_{1}=2\left(\alpha_{1}-1\right)$ and $v_{2}=2 \alpha_{2}$, Theorem 6.1 then gives the result.

\subsection{Fibrated complex contact manifolds}

The other subcategory of complex contact manifolds that motivates these results is that of fibrated complex contact manifold. A fibrated complex contact 
manifold is a complex contact manifold $Z$ foliated by leaves of $\mathscr{V}$ in such a way that a fibration is formed,

$$
R \rightarrow Z \rightarrow Z / \mathscr{V}=M
$$

In this case, $R$ is necessarily a one-dimensional complex manifold with constant curvature, so that $R$ can only be $C$, the hyperbolic plane, $S^{2}$ up to a subgroup of isometries.

A variety of fibrated complex contact manifolds are well-known. Twistorial complex contact manifolds are clearly examples of $S^{2}$-fibrated complex contact manifolds. The complex Heisenberg group is an example of a $\boldsymbol{C}$-fibration; in fact, in [10], the author has shown that one can construct a complex torus fibration for every possible dimension. More recently, Blair, Davidov and Muscarov have recently constructed a so-called hyperbolic twistor space, which gives an example with vertical leaves forming the hyperbolic plane (see [6]).

Characterizing fibrated complex contact manifolds by their vertical leaves remains an unsolved problem. In the case at hand, it is unknown whether twistor spaces over quaternionic Kähler manifolds with nonzero scalar curvature are the only $S^{2}$-fibrated complex contact manifolds. Scrutiny of the proof of Theorem 6.1 reveals that we can rewrite it as follows.

THEOREM 7.3. A three-dimensional complex manifold $(Z, J)$ is biholomorphic to the twistor space of a self-dual, Einstein four-manifold with non-zero scalar curvature if and only if $Z$ is an $S^{2}$-fibrated complex contact manifold with a bundlelike associated metric such that $\operatorname{rank}\left(\mathscr{H} \mathscr{L}_{U} J\right)=4$ for every non-zero $U \in \mathscr{V}$.

The condition regarding $\mathscr{L}_{U} J$ corresponds roughly with conditions 2 and 3 of Theorem 1.1. This makes it seem likely that there are other $S^{2}$-fibrated complex contact manifolds which are not twistorial, although the author is not aware of any such examples.

\section{REFERENCES}

[1] B. Alexandrov, G. Grantcharov and S. Ivanov, Curvature properties of twistor spaces of quaternionic Kähler manifolds, J. Geom., 62 (1998), 1-12.

[2] M. F. Atiyah, N. J. Hitchin and I. M. Singer, Self-duality in four-dimensional Riemannian geometry, Proc. Roy. Soc. London Ser. A, 362 (1978), 425-461.

[ 3 ] L. BÉRARd-Bergery, Sur de nouvelles variétés riemanniennes d'Einstein, Inst. Élie Cartan, 6 (1982), 1-60.

[4] A. L. Besse, Einstein Manifolds, Ergeb. Math. Grenzgeb. (3) 10, Springer-Verlag, Berlin, 1987.

[5] D. E. Blair, Contact Manifolds in Riemannian Geometry, Lecture Notes in Mathematics 509, Springer-Verlag, Berlin, 1976.

[6] D. E. Blair, A hyperbolic twistor space, Balkan J. Geom. Appl., 5 (2000), 9-16.

[ 7 ] W. M. Boоthby And H. C. WANG, On contact manifolds, Ann. of Math. (2), 68 (1958), 721734. 
[ 8 ] J. Davidov and O. Muskarov, On the Riemannian curvature of a twistor space, Acta Math. Hungar., 58 (1991), 319-332.

[9] B. Foreman, Variational approaches to the geometry of complex contact geometry, preprint.

[10] B. Foreman, Boothby-Wang fibrations on complex contact manifolds, Differential Geom. Appl., 13 (2000), 179-196.

[11] B. Foreman, Complex contact manifolds and hyperkähler geometry, Kodai Math. J., 23 (2000), 12-26.

[12] Y. Hatakeyama, Some notes on differentiable manifolds with almost contact structures, Osaka Math. J. (2), 15 (1963), 176-181.

[13] R. Hermann, On the differential geometry of foliations, Ann. of Math. (2), 72 (1960), 445 457.

[14] S. Ishinara, Quaternion Kählerian manifolds and fibred Riemannian spaces with Sasakian 3-structure, Kōdai Math. Sem. Rep., 25 (1973), 321-329.

[15] S. IshiHARA AND M. KonISHI, Real contact 3-structure and complex contact structure, Southeast Asian Bull. Math., 3 (1979), 151-161.

[16] S. Ishihara AND M. Konishi, Differential Geometry of Fibred Spaces, Publications of the Study Group of Geometry 8, Kyoto University, Kyoto, 1973.

[17] S. IshiHARa AND M. Konishi, Complex almost contact manifolds, Kodai Math. J., 3 (1980), 385-396.

[18] S. Ishinara and M. Konishi, Complex almost contact structures in a complex contact manifold, Kodai Math. J., 5 (1982), 30-37.

[19] S. Kobayashi, Remarks on complex contact manifolds, Proc. Amer. Math. Soc., 10 (1959), $164-167$.

[20] M. Konishi, On manifolds with Sasakian 3-structure over quaternion Kaehler manifolds, Kōdai Math. Sem. Rep., 26 (1974/75), 194-200.

[21] B. Korkmaz, Normality of complex contact manifolds, Rocky Mountain J. Math., 30 (2000), $1343-1380$.

[22] C. LeBrun, Quaternionic-Kähler manifolds and conformal geometry, Math. Ann., 284 (1989), 353-376.

[23] S. Salamon, Quaternionic Kähler manifolds, Invent. Math., 67 (1982), 143-171.

[24] A. Swann, Homogeneous twistor spaces and nilpotent orbits, Math. Ann., 313 (1999), 161188.

[25] R. O. Wells, JR., Differential Analysis on Complex Manifolds, Graduate Texts in Math. 65, Springer-Verlag, New York-Berlin, 1980.

Case Western Reserve University

Cleveland, Ohio 44106

216-368-5015

U.S.A.

(CURRENT ADDRESS)

Department of Mathematics

John CARroll University

20700 North Park Boulevard

University Heights, OHIO 44118

U.S.A.

e-mail: bforeman@jcu.edu 